\title{
A Core Group Model for Disease Transmission
}

\author{
K.P. Hadeler \\ Biomathematik \\ Universität Tübingen \\ Auf der Morgenstelle 10 \\ D-72076 Tübingen \\ Germany
}

\author{
Carlos Castillo-Chavez \\ Biometrics Unit \\ Cornell University \\ Ithaca, New York \\ USA 14853
}




\title{
A Core Group Model for Disease Transmission ${ }^{\dagger}$
}

\author{
K.P. Hadeler \\ Biomathematik \\ Universität Tübingen \\ Auf der Morgenstelle 10 \\ D-72076 Tübingen \\ Germany
}

\author{
C.Castillo-Chavez \\ Biometrics Unit \\ Cornell University \\ 332 Warren Hall \\ Ithaca, N.Y 14853-7801 \\ U.S.A.
}

\begin{abstract}
Models for sexually-transmitted diseases generally assume that the size of the core group is fixed. Publicly available information on disease prevalence may influence the recruitment of new susceptibles into highly sexually-active populations. It is assumed that the recruitment rate into the core population is low while disease prevalence is high; core group members only mix with core group members; and disease levels outside the core are negligible. It is further assumed that some core group members reduce their risk through the use of a partially effective vaccine or prophylactics.

A demographic-epidemic model is formulated where the combined size of the core and non-core population is constant. The threshold condition for an endemic infection is determined. Backward bifurcations, multiple infective stationary states, and hysteresis phenomena can be observed. Abrupt changes in disease prevalence levels are possible as a function of the success rate of the disease management program, and do not occur in the absence of such program. The general conclusion is that partially effective vaccination or education programs may increase the total number of cases while decreasing the relative frequency of cases in the core group.
\end{abstract}

\section{Introduction}

Significant changes in behavior have been observed in various homosexual populations including those of San Francisco, New York, and Boston. These changes include a reduction in average sexual activity, a decline in risky behavior, and a decrease of the rate of unprotected sexual contacts (see Baldwin and Baldwin [1], Curran et al. [5], Fineberg [7], Evans et al. [6], Martin [12], Saltzman et al. [14], Shechter et al. [15], van Griensven et al. [17],[18], Wilkenstein et al. [20], and Wiktor et al. [19]). Changes in behavior have been observed also as the result of perceived environmental risks that are measured in terms of disease prevalence (see [7] and the references therein). The combined effects of the short- and long-term

\footnotetext{
$\dagger$ This paper is dedicated in friendship to the memory of Stavros Busenberg.
} 
reduction of risk behaviors on the transmission dynamics of sexually-transmitted diseases (STDs) needs to be explored. Short-term changes may alter the course of treatable STDs such as gonorrhea or syphilis but it may have no impact on the long-term dynamics of herpes or HIV/AIDS. More importantly, the evaluation of the effectiveness of disease management strategies depends on our ability to differentiate between changes that can be traced to programmatic efforts and changes driven just by the epidemiological status of a community, e.g. knowing somebody with HIV/AIDS. Educational programs that have no significant effect in core populations with high disease prevalence may not only fail to have a positive impact in core populations with low disease prevalence but may even increase their effective group size by implying a reduction in risk within the core. Brauer et al. [3], Blythe et al. [2] have looked at the effect that disease prevalence has on the recruitment of new susceptible core-group members. Their results show that changes in behavior generate not only gradual but also qualitative differences. The core population may experience a significant reduction in size and sustained oscillations are possible. Our goal here is to look, in as simple setting as possible, at the role of partially effective disease management programs in communities where the level of disease prevalence is known (see Evans et al. [6], Martin [12], McKusick et al. [13], Shilts [16], Wiktor et al. [19])

In the study of the dynamics of sexually transmitted diseases, a population is usually subdivided into an active and relatively small core group and a weakly connected and largely inactive remainder, the non-core (see Hadeler and Müller [9] for models for general populations). In the core group one finds high transmission rates and high disease prevalence and, consequently, disease management strategies are usually aimed at the core group (see Hethcote and Yorke [11]).

The core group recruits individuals from the non-core, and the rate of recruitment may depend on the state of the core group, i.e. a high prevalence of the disease may slow down the recruitment process.

We consider a demographic-epidemic model of the simplest type where the non-core group is completely inactive. A part of the core group is educated (or vaccinated), the partially effective prophylactics (or vaccine) decreases the risk of acquiring a sexually transmitted disease. The average contact rate of the members of the core group remains constant but their risk of contracting an infection is decreased through the application of a partially effective vaccine or the use of prophylactics.

We assume that infected individuals are to some extent symptomatic and that after treatment they may return (at a constant per capita rate) either to the susceptible or to the educated (vaccinated) class.

We formulate the model as a general homogeneous system ([10], [8], [4]) although we later specialize to the case of constant population size. We determine the threshold condition for an endemic infection. An interesting feature of this model is the occurence of a backward (subcritical) bifurcation, several infected stationary states, and hysteresis phenomena, in particular abrupt changes in the 
prevalence levels of the disease are possible for small changes in the success rate of the disease management program.

\section{A simple core and non-core epidemic model}

The total population has the size $P=P(t)$, and this population is divided into the core group of size $C$ and the non-core group of size $A$, hence $P=C+A$. The population of the core group is further subdivided into susceptibles $S$, educated (vaccinated) $V$, and infected $I$, such that

$$
C=S+V+I, \quad A+C=P .
$$

The birth rate is $b>0$, the birth rate of infected is $\tilde{b} \leq b, \tilde{b} \geq 0$. The death rate is $\mu>0$, and the death rate of infected is $\tilde{\mu} \geq \mu$. The recovery rate is $\alpha+\gamma$ where $\alpha \geq 0$ is the transition rate from infected to susceptible, and $\gamma \geq 0$ is the rate of transition from infected to educated (vaccinated). The education (vaccination) rate is $\psi \geq 0$. The transmission rate from infected to susceptibles is $\beta>0$, and the transmission rate from infected to educated (vaccinated) is $\tilde{\beta}$ where $0 \leq \tilde{\beta} \leq \beta$. It is convenient to introduce $\kappa=\tilde{\beta} / \beta$. Thus $\kappa$ is the proportion of cases in which the prophylactics does not work. We assume $\kappa<1$.

Since mixing and transmission of disease takes place mainly in the core group, the incidence rates are assumed to be proportional to $I / C$ (and not to $I / P$ ). Further we assume, in this simple scenario, that the non-core does not play a direct role in the transmission process.

Recruitment into the core group is described by a function $r(I / C)$, where $r_{0}=r(0)>0$, and $r$ is decreasing in the interval $0 \leq I / C \leq 1$, that is, an increase in the prevalence slows down the rate of recruitment into the core.

The general homogeneous system reads

$$
\begin{aligned}
& \dot{A}=b(P-I)+\tilde{b} I-A r\left(\frac{I}{C}\right)-\mu A, \\
& \dot{S}=A r\left(\frac{I}{C}\right)-\beta \frac{S I}{C}-\psi S+\alpha I-\mu S, \\
& \dot{V}=\psi S-\tilde{\beta} \frac{V I}{C}+\gamma I-\mu V, \\
& \dot{I}=\frac{\beta S I+\tilde{\beta} V I}{C}-\alpha I-\gamma I-\tilde{\mu} I .
\end{aligned}
$$

This system is homogeneous of degree 1. Rather than looking for stationary solutions, we have to consider persistent (exponential) solutions of the form

$$
(A, S, V, I) \exp (\lambda t)
$$

where $\lambda$ is the exponent of demographic growth, and $(A, S, V, I)$ is the vector of (constant) proportions. For a general approach to homogeneous systems, persistent solutions and the corresponding stability theory see Hadeler [8]. 
If we insert (2) into (1) then we obtain the nonlinear eigenvalue problem

$$
\begin{aligned}
& \lambda A=b(P-I)+\tilde{b} I-A r\left(\frac{I}{C}\right)-\mu A, \\
& \lambda S=A r\left(\frac{I}{C}\right)-\beta \frac{S I}{C}-\psi S+\alpha I-\mu S, \\
& \lambda V=\psi S-\tilde{\beta} \frac{V I}{C}+\gamma I-\mu V, \\
& \lambda I=\frac{\beta S I+\tilde{\beta} V I}{C}-\alpha I-\gamma I-\tilde{\mu} I .
\end{aligned}
$$

Observe that the system (3) represents four equations for the five unknowns $A, S, V, I$ and $\lambda$. Since the vector of proportions is determined only up to a multiplicative constant, we can always add a normalization condition. Then we have five equations for five unknowns, and we can duly expect, in a nondegenerate situation, that solutions will be isolated. However, we cannot expect, that the persistent solution is unique, in general not even the infected persistent solution will be uniquely determined.

The uninfected exponential solution is easily found as

$$
\left(b, r_{0} \frac{b}{b+\psi}, r_{0} \frac{\psi}{b+\psi}, 0\right) e^{(b-\mu) t} .
$$

If we linearize the system (1) at this exponential solution then we obtain the Jacobian

$$
J=\left(\begin{array}{cccc}
b-\mu-r_{0} & b & b & \tilde{b}-b r^{\prime}(0) / r_{0} \\
r_{0} & -\psi-\mu & 0 & b r^{\prime}(0) / r_{0}+\alpha-\beta b /(b+\psi) \\
0 & \psi & -\mu & -\tilde{\beta} \psi /(b+\psi)+\gamma \\
0 & 0 & 0 & (\beta b+\tilde{\beta} \psi) /(b+\psi)-\alpha-\gamma-\tilde{\mu}
\end{array}\right)
$$

The fourth diagonal element is an eigenvalue of the matrix. According to the general theory of homogeneous systems ([8]), this eigenvalue has to be compared to the exponent of demographic growth $\lambda=b-\mu$. Thus we obtain the following stability criterion.

Result 1: The threshold condition for the stability of the uninfected exponential solution is

$$
\frac{\beta b+\tilde{\beta} \psi}{b+\psi}<(b-\mu)+\alpha+\gamma+\tilde{\mu} .
$$

If this condition is satisfied then the uninfected exponential solution is locally stable, if the strict opposite inequality holds then it is unstable.

The left hand side of (6) comprises the effects that produce infected, the left hand side those that remove infected (death and recovery, and the washout effect 
due to demographic change). We shall see in the case of population size that the threshold condition gives only partial insight into the bifurction phenomenenon.

We specialize to the case of constant population size (of the uninfected as well as of the infected population), i.e. we assume, from now on,

$$
\tilde{b}=b=\tilde{\mu}=\mu .
$$

Then the exponent of demographic growth is $\lambda=0$ in either case, and we arrive at a nonlinear system for stationary states.

For a first mathematical treatment it is convenient to normalize the size of the core group $C=1$ (and not $P=1$ ). Later we shall return to the problem of determining the size of the core group. With this normalization we have the following system of nonlinear equations,

$$
\begin{aligned}
& 0=\mu(1+A)-A r(I)-\mu A, \\
& 0=A r(I)-\beta S I-\psi S+\alpha I-\mu S, \\
& 0=\psi S-\tilde{\beta} V I+\gamma I-\mu V, \\
& 0=\beta S I+\tilde{\beta} V I-\alpha I-\gamma I-\tilde{\mu} I,
\end{aligned}
$$

together with

$$
S+V+I=1 .
$$

First we look for uninfected stationary states, $I=0$. Then

$$
\begin{aligned}
& 0=\mu(1+A)-r_{0} A-\mu A, \\
& 0=r_{0} A-\psi S-\mu S, \\
& 0=\psi S-\mu V,
\end{aligned}
$$

from where we find immediately the unique uninfected stationary state (see also (4))

$$
\left(A_{0}, S_{0}, V_{0}, I_{0}\right)=\left(\frac{\mu}{r_{0}}, \frac{\mu}{\mu+\psi}, \frac{\psi}{\mu+\psi}, 0\right)
$$

(observe $S_{0}+V_{0}=1$ ). For an infected solution $I>0$, we have (8) and

$$
\begin{aligned}
& 0=\mu(1+A)-A r(I)-\mu A, \\
& 0=A r(I)-\beta S I-\psi S+\alpha I-\mu S, \\
& 0=\psi S-\tilde{\beta} V I+\gamma I-\mu V, \\
& 0=\beta S+\tilde{\beta} V-\alpha-\gamma-\mu .
\end{aligned}
$$

$¿$ From equation (10a) we get the relation

$$
\operatorname{Ar}(I)=\mu
$$


which can be used to replace the nonlinearity $r(I)$ in the remaining equations,

$$
\begin{aligned}
& 0=\mu-\beta S I-\psi S+\alpha I-\mu S, \\
& 0=\psi S-\tilde{\beta} V I+\gamma I-\mu V, \\
& 0=\beta S+\tilde{\beta} V-\alpha-\gamma-\mu .
\end{aligned}
$$

Later we use equation (11) to compute the quantity $A$. Here we use (12b)(12c) to eliminate the variable $V$ and obtain an equation for $S$,

$$
S=\frac{(\alpha+\mu)(\tilde{\beta} I+\mu)+\gamma \mu}{\beta \tilde{\beta} I+\beta \mu+\tilde{\beta} \psi} .
$$

¿From (12a) we find

$$
S=\frac{\mu+\alpha I}{\beta I+\mu+\psi}
$$

and thus

$$
\frac{\mu+\alpha I}{\beta I+\mu+\psi}=\frac{(\alpha+\mu)(\tilde{\beta} I+\mu)+\gamma \mu}{\beta \tilde{\beta} I+\beta \mu+\tilde{\beta} \psi} .
$$

Multiplying by the denominators, we arrive at a quadratic equation for the infection level $I$,

$$
(\beta I+\mu+\psi)[(\alpha+\mu)(\tilde{\beta} I+\mu)+\gamma \mu]-(\alpha I+\mu)(\beta \tilde{\beta} I+\beta \mu+\tilde{\beta} \dot{\psi})=0 .
$$

After collecting terms we find that $I$ is a zero of the function

$$
\begin{gathered}
f(I)=\beta \tilde{\beta} I(I-1)+\beta I(\mu+\gamma)+\tilde{\beta} I(\mu+\alpha+\psi) \\
-\beta \mu-\tilde{\beta} \psi+(\mu+\alpha+\gamma)(\mu+\psi) .
\end{gathered}
$$

For a given set of parameters, the zeros $I \in(0,1)$ of this function correspond to feasible infected stationary states. From $f(1)>0, f^{\prime}(1)>0$ it follows that $f$ has no zeros in $[1, \infty)$. There is a single root in $(0,1)$ if and only if $f(0)<0$, i.e. if

$$
\beta \mu+\tilde{\beta} \psi>(\mu+\alpha+\gamma)(\mu+\psi) .
$$

This important inequality can be reformulated in several ways.

We introduce the basic reproduction number (in the absence of education)

$$
R_{0}=\frac{\beta}{\mu+\alpha+\gamma}
$$

and also the reproduction number in case everybody is in the educated class,

$$
\tilde{R}_{0}=\frac{\tilde{\beta}}{\mu+\alpha+\gamma}=\kappa R_{0}
$$


Then we introduce the reproduction number in the presence of the prophylactics level $\kappa$ and the education rate $\psi$,

$$
R(\psi, \kappa)=\frac{\mu}{\mu+\psi} R_{0}+\frac{\psi}{\mu+\psi} \tilde{R}_{0}=\frac{\mu+\kappa \psi}{\mu+\psi} R_{0} .
$$

We see that $R(\psi, \kappa)$ is a decreasing function of $\psi$, and an increasing function of $\kappa$. The inequality (18) is equivalent with $R(\psi, \kappa)>1$. We state the threshold condition as follows.

Result 2: The inequality $R(\psi, \kappa)<1$ is the threshold condition for the stability of the uninfected stationary state. If it is satisfied then the uninfected stationary state is locally stable, and there is either no infected state or there are two infected stationary states. If $R(\psi, \kappa)>1$ is satisfied then the uninfected stationary state is unstable and there is exactly one infected stationary state.

The most interesting case occurs when $R_{0}>1$ (the disease can spread in an uneducated population) and $\tilde{R}_{0}<1$ (the disease cannot spread in totally educated population). In this case increasing $\psi$ will lead to values $R(\psi, \kappa)<1$ and thus to transition to another situation. Whereas in classical epidemic models the condition $R(\psi, \kappa)<1$ will ensure the elimination of the disease, the possible transitions in the present model are more complicated.

When the parameters are varied then the uninfected state can change from a stable situation to an unstable situation in the form of a bifurcation. Quite in contrast to usual single group epidemic models there may be a backward bifurcation (the bifurcating infected solution is unstable) which we shall investigate now.

The case of two feasible infected stationary states occurs when $f^{\prime}(0)<0$, i.e. $R(\psi, \kappa)<1$, and the minimum of $f$, assumed at some point in $(0,1)$, is negative. One can write down the correponding inequalities explicitly, but they do not give much insight.

Therefore we take another approach. In equation (16) we solve for $\psi$ and express $\psi$ as a fuction of $I$,

$$
\psi(I)=R_{0}(1-\kappa) \frac{\alpha I+\mu}{1-R_{0} \kappa(1-I)}-(\mu+\alpha+\gamma) R_{0} I-\mu .
$$

If we keep all parameters (except $\psi$ ) fixed, and let $I$ run from 0 to 1 , then we obtain for each infection level the corresponding education rate. In particular we find the critical education level

$$
\psi(0)=\psi^{*}=\frac{\mu\left(R_{0}-1\right)}{1-R_{0} \kappa} .
$$

Result 3: It takes at least the education rate $\psi^{*}$ in order to achieve $I=0$. 
For $\psi=0$ we get the equation

$$
R_{0}(1-\kappa) \frac{\alpha I+\mu}{1-R_{0} \kappa(1-I)}=(\mu+\alpha+\gamma) R_{0} I+\mu .
$$

The right hand side is a straight line running from $\mu$ to $(\mu+\alpha+\gamma) R_{0}+\mu$. The left hand side is a hyperbola (concave or convex) running from $R_{0}(1-\kappa) \mu /\left(1-R_{0} \kappa\right)>$ $\mu$ to $R_{0}(1-\kappa)(\mu+\alpha)<(\mu+\alpha+\gamma) R_{0}+\mu$. Thus we get, as expected, uniqueness.

Result 4: In the absence of education the infected stationary state is uniquely determined.

We return to (22). From

$$
\psi^{\prime}(I)=R_{0}(1-\kappa) \frac{\alpha-(\mu+\alpha) \kappa R_{0}}{\left[1-R_{0} \kappa(1-I)\right]^{2}}-R_{0}(\mu+\alpha+\gamma)
$$

it follows that the function $\psi^{\prime}(I)$ has at most two zeros. Furthermore we see the following property.

Result 5: Let $\alpha \leq \kappa R_{0}(\alpha+\mu)$ (in particular $\alpha=0$ ). Then $\psi(I)$ is decreasing.

Thus $\psi(I)$ is decreasing from the positive value $\psi^{*}=\psi(0)$, it stays positive in the interval $0 \leq I<I_{0}$ where $I_{0}$ is the prevalence at $\psi=0$, then becomes negative. In other words, an increase of the education effort $\psi$ always leads to a decrease of the proportion of infected in the core group. This behavior occurs in particular in the case $\alpha=0$, i.e. when infected, after recovery, do not return to the uneducated class.

For other parameter sets, the derivative $\psi^{\prime}(0)$ may be positive, we see the following.

\section{Result 6: If}

$$
\frac{\alpha-(\mu+\alpha) R_{0} \kappa}{\left(1-R_{0} \kappa\right)^{2}}>\frac{\mu+\alpha+\gamma}{1-\kappa}
$$

then there is a backward bifurcation, i.e. there is a branch of stationary solutions with the following behavior. If $\psi$ is increased from the critical level $\psi^{*}$ then an initially small proportion of infected $I$ in the core group will increase rather than decrease.

Of course, the solutions presented in Result 6 are unstable. Nevertheless there is an interesting hysteresis phenomenon near $I=0, \psi=\psi^{*}$. Assume the system is in the stable uninfected state with some $\psi>\psi^{*}$. If $\psi$ is decreased below $\psi^{*}$ then the uninfected state becomes unstable and the system jumps to an infected state with a large proportion of infected. If $\psi$ is increased again then the prevalence stays at the high level unless $\psi$ becomes very large when the system jumps down to the uninfected state. 
So far we have worked from the inside, i.e. from within the core group. For a core group normalized to size 1 we have determined the possible stationary proportions of infected, which are $\tilde{I}=0$ and possibly one or two non-zero prevalence levels $\tilde{I}_{1}, \tilde{I}_{2}$. For each of these levels one can use equation (11) to determine the size of the corresponding non-core $A$ as

$$
A=\mu / r(\tilde{I})
$$

and the total population size $P=1+A$ that would correspond to this prevalence $\tilde{I}$ in a core group of size 1 .

In reality, however, the total population size $P$ is given, and for given parameters the size of the core group $C=S+V+I$ is intrinsically determined. There may be even several stationary core group sizes. Thus we have to proceed as follows.

Let $P$ and the model parameters be given. From the model parameters we can determine $\tilde{I}$ (i.e. $\tilde{I}=0$, and possibly $\tilde{I}_{1}, \tilde{I}_{2}$ ). Then the actual size of the core group is

$$
C=\frac{\operatorname{Pr}(\tilde{I})}{\mu+r(\tilde{I})} .
$$

Thus to each value of the proportion of infected there is an actual size of the core group. Since $r$ is a decreasing function (and $x \mapsto x /(\mu+x)$ is increasing) we find the following monotonicity property.

Result 7: The size of the core group is a decreasing function of the proportion of infected within the core group.

Thus, if one considers an isolated stationary solution and parameters are varied then the proportion of infected is decreasing while the core group is increasing. This may look like a simple dilution effect but it is fact more complicated.

Indeed, the number of infected cases is given by

$$
I=C \tilde{I}=\frac{\operatorname{Pr}(\tilde{I}) \tilde{I}}{\mu+r(\tilde{I})} .
$$

Thus the relation between the infected proportion within the core and the size of the core is governed by the monotone function (28) while the relation between the infected proportion and the total number of infected is given by the function (29) which need not be decreasing.

We illustrate the various possibilities with an example,

$$
r(\tilde{I})=r_{0}(1-b \tilde{I})_{+} .
$$

For $\tilde{I} \geq 0$, the function (29) is first increasing, then decreasing, and finally becomes zero. The parameters can be chosen such that the maximum falls into the interval 
$(0,1)$. Then the function $(29)$ is not monotone. Hence the question whether the total number of cases goes up or down as a function of the education strategy will depend on the relative position if the zero(s) of $f$ with respect to the said maximum.

In Fig.1 we present the graph of the function $f(\tilde{I})$ for a fixed set of parameters $\mu=0.2, \alpha=4, \gamma=0.1, \beta=6, \tilde{\beta}=3$. Then $R_{0}=1.4, \tilde{R}_{0}=0.7$. The parameter $\psi$ is ranging from 0 to 0.4 in steps of 0.08 . The graph moves up as $\psi$ increases. For $\psi=0$ and for small values of $\psi$ the function $f$ has one zero in $(-\infty, 0)$ and one in $(0,1)$, the latter corresponding to the unique infected stationary solution. For $\psi$ at about 0.24 both solutions become feasible. The prevalence $\tilde{I}$ at the upper solution is reduced in comparison to $\psi=0$, but there is also the lower (unstable) infected solution. For still larger values of $\psi$ these solutions coalesce and disappear, leaving the uninfected solution $(\tilde{I}=0)$ as the only stationary state.

In Fig. 2 we show the bifurcation diagram $(\tilde{I}$ versus $\psi$ ) for the same set of parameters, except for $\tilde{\beta}$, which ranges from 0 to 3.3 in steps of $0.3\left(\tilde{R}_{0}\right.$ from 0 to 0.77 ). The graphs move to the right as $\tilde{\beta}$ increases. For small $\tilde{\beta}$ the graph is monotone, in particular $\psi^{\prime}(0)<0$. As $\tilde{\beta}$ is increased, $\psi^{\prime}(0)$ becomes positive and the graphs are bent. One sees the backward bifurcation at $\tilde{I}=0$ and the "knee" of the saddle-node bifurcation.

In Fig.3, for the same set of parameters and the recruitment function (30) with $r_{0}=0.1$ and $b=3$, the total number of infected $I$ is plotted against the value of $\psi(\mathrm{P}=1)$. Only the first $(\tilde{\beta}=0)$ and the last curve $(\tilde{\beta}=3.3)$ are presented. The backward bifurcation near $I=0$ persists since the function $r$ is approximately linear near $\tilde{I}=0$. Since the function $r$ is not monotone, the graph is not monotone near $\psi=0$. This example shows a situation where an education campaign with low efficiency (small positive $\psi$ as compared to $\psi=0$ ) has a detrimental effect: Although the relative proportion of infected $\tilde{I}$ in the core group is decreased, the size of the core group is increased and the total number of infected $I$ is going up.

\section{Conclusions}

Unfortunately, it appears that a population at risk of STDs needs to experience relatively high levels of disease prevalence before changes in behavior help stem disease spread (see Fineberg [7]). Our results illustrate the potential for a sad and somewhat paradoxical situation; the use of prophylactics or a partially effective vaccine may increase the size of the core group relative to what "it would have been in the absence of any disease management program. These increases on the effective size of the core population may generate unexpected and abrupt changes in disease levels.

If higher disease levels lead to the recruitment of fewer individuals to highly sexually active groups then a reduction in risk through a partially effective vaccine or the use of prophylactics, by decreasing prevalence, may in fact increase the size 
of the network. Furthermore, the evaluation of a disease managment program through the random sampling from the core group may yield misleading results as the size core changes. In fact it is possible to observe a reduction in prevalence in the core group while the actual number of cases is increased.

Evaluation of control programs must take into account the effects of differential recruitment, disease-induced mortality, and the actual size of the infected class. The availability of partially effective risk-reducing mechanisms may in fact increase the size and number of highly-active sexual groups and, possibly, the number of cases. Because populations are not isolated, disease management programs that do not have as one of their main objectives the promotion of substantial and permanent behavioral changes through counseling and other means may, in the long run, increase the number of cases even in moderately active sexual networks. To put it succinctly, programs that do not include effective counseling services may cause unnecessary risks to populations with low average levels of sexual activity.

Acknowledgements: This research was partially supported by NSF grant DEB925370 (Presidential Faculty Fellowship Award) and by the U.S. Army Research Office through the Mathematical Sciences Institute of Cornell University (contract DAAL03-91-C-0027) to C.C.-C. and by a SIMS (NIDA) grant to K.P.H. This work was done while C.C.-C. visited Tübingen University during his stay at the Isaac Newton Institute.. 


\section{References:}

[1] Baldwin J.D., Baldwin, J.I., Factors affecting AIDS-related sexual risk-taking behavior among college students. J. Sex Research 25, 181-196 (1988)

[2] Blythe, S. P., Brauer, F., Castillo-Chavez, C., Velasco-Hernández, J.X., Sexually transmitted diseases with recruitment. Biometrics Unit Technical Report BU-1193-M), Cornell University (1993)

[3] Brauer, F., Blythe, S.P., Castillo-Chavez, C., Demographic recruitment in sexually transmitted disease models. Biometrics Unit Technical Report BU1154-M, Cornell University (1992)

[4] Busenberg, S., Hadeler, K.P., Demography and epidemics. Math. Biosc. 101, 63-74 (1990)

[5] Curran, J. W., Jaffe, H.W., Hardy, A.M., Morgan, M.W., Selik, R.M., Dondero, T.J., Epidemiology of infection and AIDS in the United States. Science 293, 610-616 (1988)

[6] Evans, B. A., McLean, K.A., Dawson, S.G., Trends in sexual behavior and risk factors for the HIV infection among homosexual men, 1984- 1987. Brit. Med. J. 298, 215-218 (1989)

[7] Fineberg, H. V., Education to prevent AIDS: prospects and obstacles. Science 239, 592-596 (1988)

[8] Hadeler, K.P., Periodic solutions of homogeneous equations. J.Diff.Equ. 95, 183-202 (1992)

[9] Hadeler, K.P., Müller, J., Vaccination stategies for sexually transmitted diseases. In: Arino, O., Axelrod, D., Kimmel, M. (eds.) Proc. Conf. Population Dynamics, Pau (France) 1992 (1994)

[10] Hadeler, K.P., Waldst tter, R., W rz-Busekros, A., Models for pair formation in bisexual populations. J. Math. Biol. 26, 635-649 (1988)

[11] Hethcote, H. W., Yorke, J.A., Gonorrhea: Transmission Dynamics and Control. Lecture Notes in Biomathematics 56, Springer-Verlag 1984

[12] Martin, J. L., The impact of AIDS in gay male sexual behavior patterns in New York City. Am. J. of Pub. Health 77, 578-581 (1987)

[13] McKusick, L., Wiley, J.A., Coates, T.J., Stall, R., Saika, B., Morin, S., Hortsman, C.K., Conant, M.A., Reported changes in the sexual behavior of men at risk for AIDS, San Francisco, 1983-1984: the AIDS behavioral research project. Public Health Reports 100, 622-629 (1985)

[14] Saltzman, S.P., Stoddard, A.M., McCusker, J., Moon, M.W., Mayer, K.H., Reliability of self-reported sexual ehavior risk factors for HIV in homosexual men. Public Health Report 102, 692- 697 (1987)

[15] Shechter, M.T., Craib,, K.J.P., Willoughby, B., Patterns of sexual behavior and condom use in a cohort of homosexual men. Amer. J. Publ. Health 78, 1535-1538 (1988) 
[16] Shilts, R., And the band played on. San Martin's press, New York 1987

[17] van Griensven, G.J.P., de Vroome, E.M.M, Goudsmit, J., Coutinho, R.A., Changes in sexual behavior and the fall in incidence of HIV infection among homosexual men. Br. Med.J. 298, 218-221 (1989)

[18] van Griensven, G.J.P, de Vroome, E.M.M., Tielman, R.A.P., Effect of human immuno-deficiency virus (HIV) antibody knowledge on high-risk sexual behavior with steady and non-steady partners among homosexual men. Am.J. Epidem. 129, 596-603 (1989)

[19] Wiktor, S.Z., Biggar, R.J., Melby, M., Ebbesen, P., Colclough, G., Di Gioia, R., Sanchez, W.C., Grossman, R.J., Goedert, J., Effects of knowledge of human immunodeficiency virus infection status on sexual activity among homosexual men. J.of AIDS 3, 62-68 (1990)

[20] Wilkenstein, W. Jr., Wiley, J.A., Padian, N.S., The San Francisco Men's Health Study, continued decline in HIV seroconversion rates among homosexual/bisexual men. Am. J. Public Health 78, 1472-1474 (1988) 


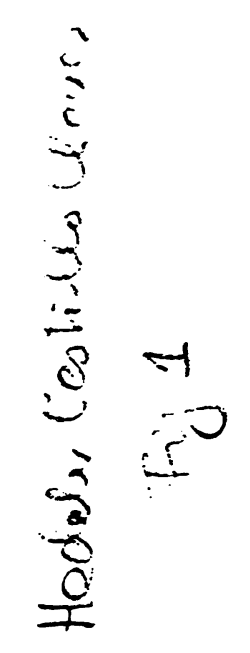

.

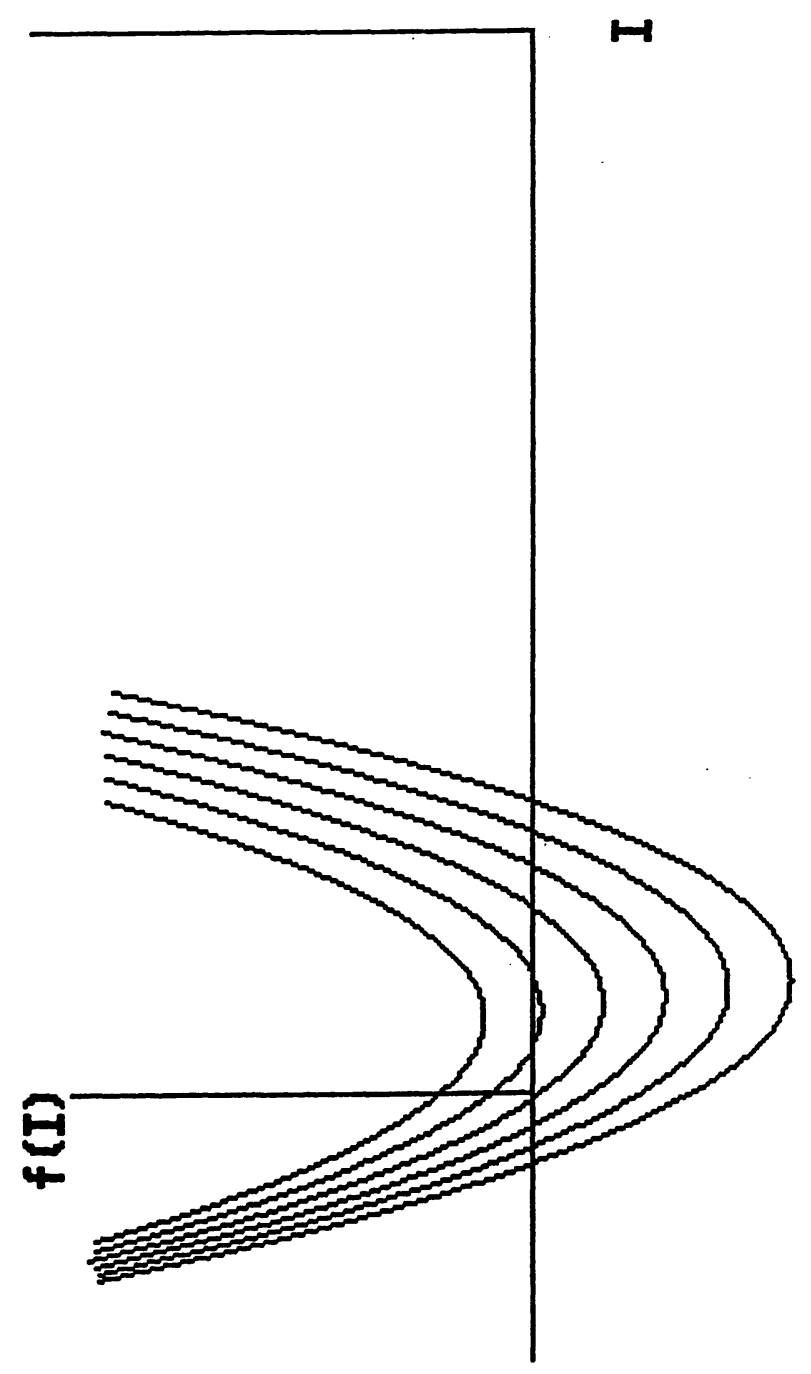



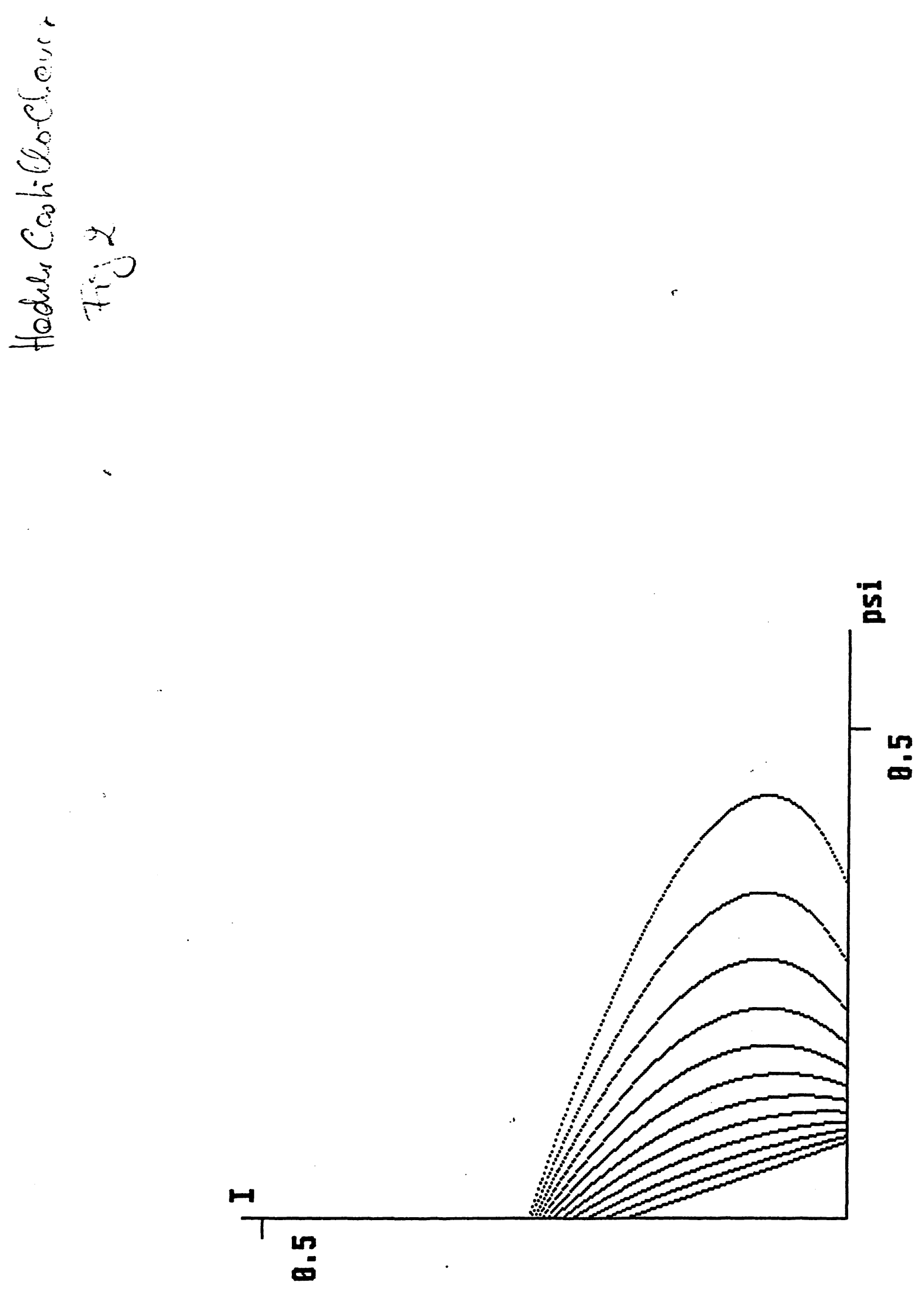


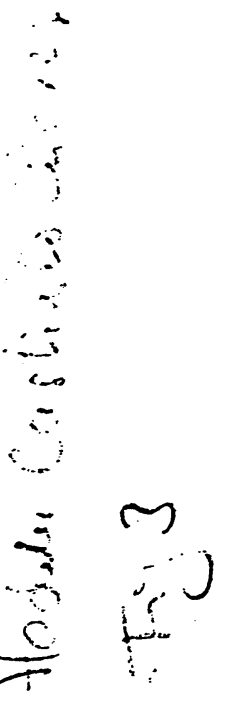

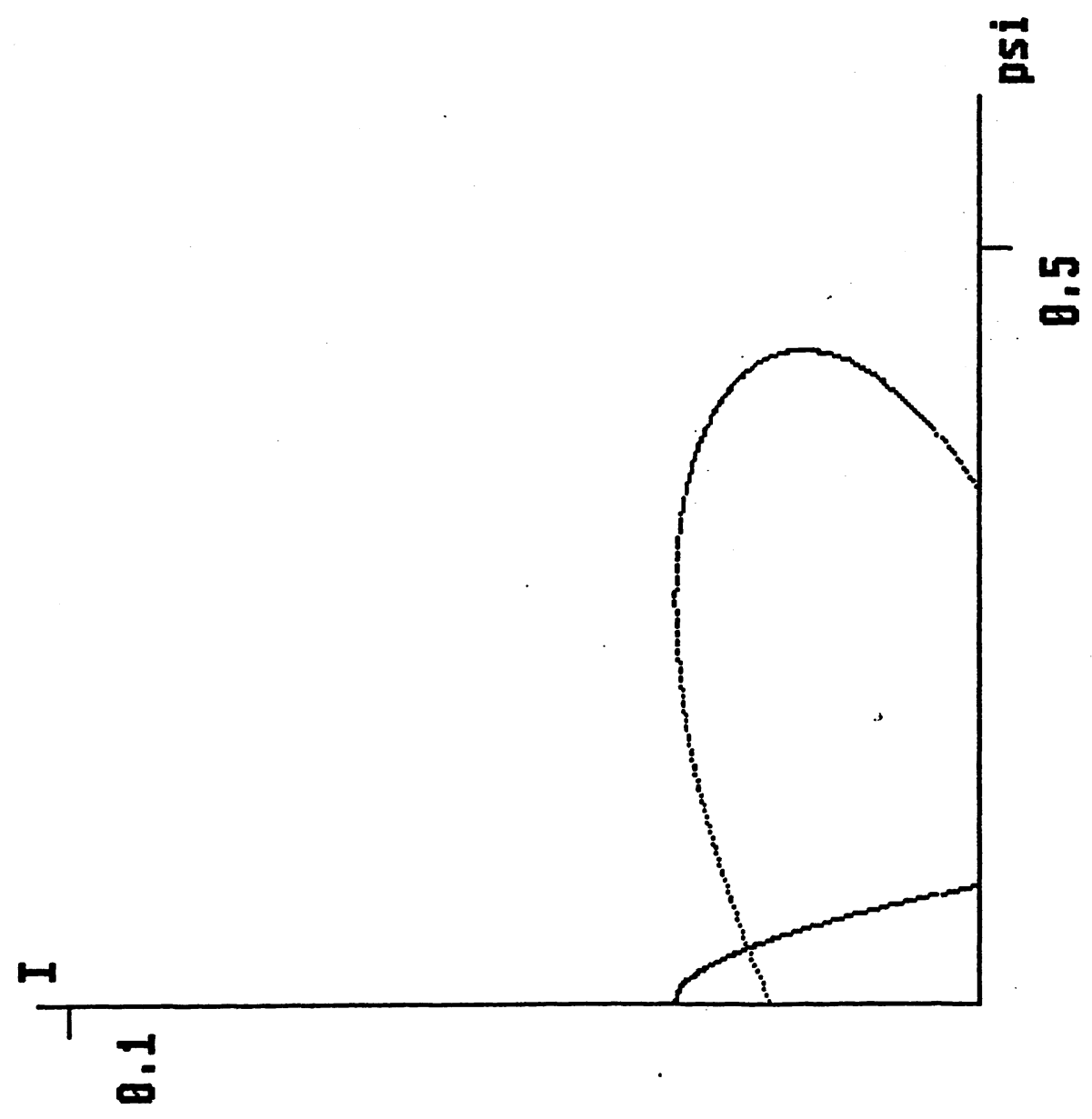

